\title{
MARS: a tool for haplotype-resolved population-based structural variation detection
}

\author{
Lu Zhang ${ }^{1}$, Arend Sidow ${ }^{2}$, and Xin Zhou ${ }^{3,4 *}$ \\ ${ }^{1}$ Department of Computer Science, Hong Kong Baptist University, Kowloon Tong, Hong Kong, ${ }^{2}$ Department of Pathology, Stanford \\ University, Stanford, CA 94305, USA, ${ }^{3}$ Department of Biomedical Engineering, Vanderbilt University, Nashville, TN 37235, USA, \\ ${ }^{4}$ Department of Computer Science, Vanderbilt University, Nashville, TN 37235, USA \\ *To whom correspondence should be addressed. \\ Associate Editor: $\mathrm{XXXXXXX}$ \\ Received on XXXXX; revised on XXXXX; accepted on XXXXX
}

\begin{abstract}
Motivation: Linked-reads enables genome-wide phased diploid assemblies. These haplotype-resolved assemblies allow us to genotype structural variants (SVs) with a high sensitivity and be able to further phase them. Yet, existing SV callers are designed for haploid genome assemblies only, and there is no tool to call SV from a large population of diploid assemblies which can define and refine SVs from a global view.

Results: We introduce MARS (Multiple Alignment-based Refinement of Svs) in linked-reads for the detection of the most common SV types - indels from diploid genome assemblies of a large population. We evaluated SVs from MARS based on Mendelian law of inheritance and PacBio HiFi reads and it achieved a high validation rate around $73 \%-87 \%$ for indels that we have selected from 34 assembled samples.
\end{abstract}

Availability: Source code and documentation are available on https:// qithub.com/maiziex/MARS. Contact: maizie.zhou@vanderbilt.edu

Supplementary information: Supplementary data are available at Bioinformatics online.

\section{Introduction}

The ultimate goal of diploid human genome sequencing is to obtain a comprehensive, and ideally $100 \%$ accurate, list of phased genetic variation harbored by each individual's 'germline' genome (Levy et al., 2007). Genetic variation originates via a multitude of molecular mechanisms and takes many forms, including single nucleotide variants (SNPs) and the most common types of structural variants (SVs), insertions and deletions (indels). Linked-read sequencing has recently emerged as a potentially cost-effective solution for genome reconstruction and variation discovery. It is based on the idea that barcoded short reads can be generated from original long fragments of the DNA preparation such that all short reads from the same fragment have the same barcode. The approach was pioneered by 10x Genomics (Zheng et al., 2016). Even though their linkedreads has been discontinued recently, several companies have put effort into this promising technology, such as single tube long fragment reads (stLFR) by BGI (Wang et al., 2019), TELL-Seq by Universal Sequencing Technology (Chen et all, [2020), etc. Linked-read sequencing has low base pair-level error rates, and if the data are leveraged appropriately with custom algorithms, they facilitate discovery of SNPs and indels with good accuracy, as well as long-range haplotyping.
Regardless of technology employed, cost-effective and accurate indel discovery in personal genomes is still a challenge. While benchmarking standards have been developed for a handful of reference genomes, the confidence in each individual indel call is difficult to estimate (Chapman et all, 20120; Zook et al, 20120). An additional challenge is the precise and accurate determination of breakpoints, which is sometimes furthe complicated by compound events such as insertions that also delete short sequences around the insertion site (Cameron et all, 2019). This scenario could be pretty complicated and messy when calling SVs for a large population. A final complication is the fact that the molecular mechanism that gave rise to the indel allele is often the opposite of the call, whenever the reference genome carries the derived allele and the individual's sequenced genome has the ancestral allele.

We here address some of these challenges by taking a multiple alignment-based approach to inform accuracy and precision of inde discovery through a tool called MARS. We performed reference-assiste whole genome diploid assembly of 34 linked-read genomes using Aquila, discovered indels ( $>20 \mathrm{bp}$ by default) in each assembled genome, an then assessed reliability of our calls on the basis of multiple sequence alignments of the indels and surrounding sequence. 


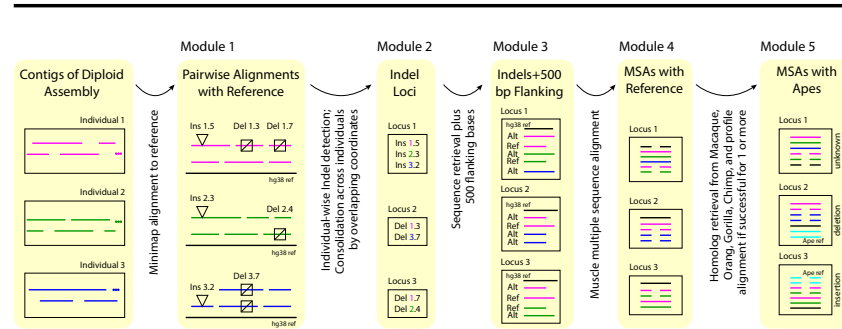

Fig. 1. Schematic diagram of the last MSA module. The detailed descriptions are in the methods section

\section{Methods}

MARS is a haplotype-resolved population-based structural variation detection method for linked-read diploid assemblies. The input files are whole genome diploid contigs from each sample. Aquila (Zholl et al, (20) or Aquila_stLFR (Lill et al, 202I) can be used to assemble whole genome diploid contigs on 10x and stLFR linked-reads data, respectively. In this paper, we have assembled 34 samples of 10x linked-reads data with Aquila (see details in Supplementary Information).

The first module of MARS performs a pairwise contig-to-reference comparison to generate genome-wide variant calls from each haplotype independently. It then merges variants from both haplotypes to generate diploid variant calls. This step is performed for each sample. In the second module, MARS merges SVs of all samples through overlapping coordinates and a hard threshold ( $1 \mathrm{bp}$ for deletion and 100bp for insertion). The third module extracts haplotype-resolved sequences from the samples that contribute their original SVs to the merged SVs. To extract the haplotype-resolved sequences around the SV for each sample, it refers back to breakpoints of the original SV and extracts the SV sequence plus the left and right $500 \mathrm{bp}$ flanking regions from the assembled haplotype-resolved contigs. There are two scenarios to extract the haploid sequences for each sample. If the SV is homozygous or compound-heterozygous, MARs extracts both haploid sequences simply based on the breakpoints of the original SV from the sample. If the SV is heterozygous, it needs to extract the haploid sequence covering the reference allele and the haploid sequence covering the actual SV. For each merged SV, it also extracts the human reference sequence based on the breakpoints of the merged SV. Because the breakpoints of the new merged SV span the breakpoints of all original SVs, the reference sequence it extracts enclose all individual haploid sequences. In the fourth module, MARS uses MUSCLE (Edgar, 2004) to perform multiple sequence alignment (MSA) of the reference sequence and al extracted haplotype-resolved sequences. It then scans through all MSAs to determine the outer breakpoints of each new merged SV from a global scale. To detect the breakpoints, it discovers the first large gap ( $\geq 20 \mathrm{bp})$ on all sequences from left and right side, respectively. An optional fifth module of MARS facilitates investigation of the ancestral state of indels, by profile-aligning the homologous portions of Apes reference sequences (for example, Orangutan, Chimpanzee, Macaque or Gorilla) to each MSA

\section{Results and Discussion}

We collected the linked-reads from publicly available 26 samples and sequenced 8 additional samples (Supplementary Note and Supplementary Table 1) to evaluate the performance of MARS. We selected SVs supported by at least two samples for later analyses. We adopted two strategies to validate SV calls: Mendelian inheritance and PacBio HiFi reads (Supplementary Note). For inheritance, the average validation rates were $84.48 \%$ and $78.92 \%$ for deletions and insertions (Supplementary Table 2) based on the four trios in our samples (Supplementary Table 3); for the four samples with PacBio HiFi reads, an average of $73.03 \%$ of deletions and $86.66 \%$ of insertions (Supplementary Table 4) were validated. The overall AUROC (Area under the ROC Curve) based on the SV confidence scores was 0.848 by applying ten-fold cross validation to the SVs validated by PacBio HiFi reads.

We also annotated the SVs functionally with Ensembl Varian Effect Predictor (McLaren et al, (2016) (Supplementary Figure 1-5) and RepeatMasker (Tarailo-(iraovac and Chen, 2009) (Supplementary Figure 6). Coding SVs were rarely in repeat sequences and their lengths were commonly a multiple of three, suggesting the SV breakpoints refined by MARS were accurate. The performance of the SV confidence scores was a little bit different across the SVs located in the different functional regions (Supplementary Figure 7). We further selected derived deletions and insertions supported by different large numbers of haplotype-solved sequences; consistent with evolutionary expectation, the vast majority of full length Alu sequences were derived insertions.

To the best of our knowledge, MARS is the first tool to detect and refine SVs from a large population of diploid assemblies in linked-reads, and this approach could be further applied to long reads in the near further.

\section{References}

Cameron, D. L. et al. (2019). Comprehensive evaluation and characterisation of short read general-purpose structural variant calling software. Nat Commun, 10(1), 3240.

Chapman, L. M. et al. (2020). A crowdsourced set of curated structura variants for the human genome. PLoS Comput Biol, 16(6), e1007933.

Chen, Z. et al. (2020). Ultralow-input single-tube linked-read library method enables short-read second-generation sequencing systems to routinely generate highly accurate and economical long-range sequencing information. Genome Res, 30(6), 898-909.

Edgar, R. C. (2004). MUSCLE: a multiple sequence alignment method with reduced time and space complexity. BMC Bioinformatics, 5, 113

Levy, S. et al. (2007). The diploid genome sequence of an individual human. PLoS Biol, 5(10), e254.

Liu, Y. H. et al. (2021). Aquila_stLFR: diploid genome assembly based structural variant calling package for stLFR linked-reads. Bioinformatics Advances. vbab007.

McLaren, W. et al. (2016). The Ensembl Variant Effect Predictor. Genome Biol, 17(1), 122.

Tarailo-Graovac, M. and Chen, N. (2009). Using RepeatMasker to identify repetitive elements in genomic sequences. Curr Protoc Bioinformatics, Chapter 4, Unit 4.10.

Wang, O. et al. (2019). Efficient and unique cobarcoding of secondgeneration sequencing reads from long DNA molecules enabling costeffective and accurate sequencing, haplotyping, and de novo assembly. Genome Res, 29(5), 798-808.

Zheng, G. X. et al. (2016). Haplotyping germline and cancer genomes with high-throughput linked-read sequencing. Nat Biotechnol, 34(3), 303-311.

Zhou, X. et al. (2021). Aquila enables reference-assisted diploid persona genome assembly and comprehensive variant detection based on linked reads. Nat Commun, 12(1), 1077.

Zook, J. M. et al. (2020). A robust benchmark for detection of germline large deletions and insertions. Nat Biotechnol, 38(11), 1347-1355. 